\title{
EVOLUÇÃO MORFOLÓGICA E TEXTURAL DE DIAMANTES BASEADA NO MÉTODO DA FOTOGONIOMETRIA
}

\author{
Maximiliano Martins ${ }^{1}$, Vladimir Rakin ${ }^{2}$, Vitaly Petrovsky², Joachim Karfunkel ${ }^{3}$, \\ Alexander Sukharev ${ }^{2} \&$ Vassily Filippov ${ }^{2}$
}

\begin{abstract}
The curvilinear surfaces of 47 diamonds from Macaúbas river basin (MG) were studied on the parabolic goniometer. For the diamond description primary genetic value is given to the form of the crystal, but the secondary is given to the sculpture of the surface. Crystallographic terms: octahedroid, dodecahedroid, cuboid are usually used for classification of the form of the diamonds. For practical purposes this is possible to consider reasonable. To determine the figure of the curve-faced crystal, the main indicator is the position of maximum of intensity of reflected light on the stereographic projections of crystal, but not angular sizes of the reflex. Curvilinear faces of diamonds suggest their dissolution processes. The symmetry of crystal shape $\mathrm{O}_{h}$ for such diamonds usually decreases to $\mathrm{C}_{2 h}$, $\mathrm{C}_{3 \mathrm{~h}}$ or $\mathrm{C}_{4 \mathrm{~h}}$, which explains a crystal rotation at the dissolution according to the $\mathrm{P}$. Curie principle. The curvilinear surfaces of the diamond crystals are possible to describe by means of fragments of the surfaces of triaxial ellipsoid duplicated with symmetry group $\mathrm{O}_{\mathrm{h}}$ up to twelve. Ellipsoid have semiaxis A1, A2, A3, numbered in the order of their growth, and well-ordered relatively to the crystal structure. Axis A2 is always directed along the axis L4, but two other axes are inclined to axis L2 under the small angle (up to 7 degrees). Angle á defines an appearance of so called "face-seam" on the surfaces of the diamond. There are 24 curvilinear surfaces formed the dodecahedroid with "faceseams", as a limiting shape of the diamond dissolution. Ellipsoidal surface of the diamond is the dynamic indicator of stability of the crystal structure of a homoeopolar crystal to the processes of dissolution and partly detrition. The existence of both a triangular etching pits with the flat bottom on the surfaces (111) and a negative relief of the surface for all directions $\langle 110\rangle$ shows a regeneration process of a diamond.
\end{abstract}

Keywords: diamond, goniometry, dissolution, ellipsoid, dodecahedroid

\section{MORFOLOGIAEXTERNAEELEMENTOSDE SUPERFÍCIE NOSDIAMANTES: CONSIDERAÇÕES GERAIS}

O diamante cristaliza-se no sistema isométrico, no grupo espacial $\mathrm{Fd} 3 \mathrm{~m}\left(\mathrm{a}=3.57^{\circ} \mathrm{A}\right)$. As formas mais comuns resultantes da cristalização do diamante são: i) octaedro $\{111\}$, ii) cubo $\{100\}$, iii) formas combinadas (octaedro+dodecaedro+cubo) ou iv) geminações segundo a rotação em torno de um eixo de simetria (comumente a geminação desenvolvida segundo a lei do espinélio). Outras formas são o rombododecaedro $\{110\}$, icositetraedro (24 faces trapezoidais), trioctaedro (24 faces triangulares) e hexaocatedro (48 faces). Todas estas formas são caracterizadas por superfícies planas, com desenvolvimento de estruturas em degraus positivos na face octaédrica e arestas retilíneas (Kukharenko 1954).

Contudo, um número expressivo de diamantes exibe morfologia externa abaulada (rounded diamonds), cujo desenvolvimento possivelmente estaria relacionado aos processos de dissolução química do cristal ocorridos concomitantemente com os estágios de formação do diamante e/ou posteriores a este. Estes processos resultariam na modificação das superfícies planas de crescimento das faces cúbicas, octaédricas ou dodecaédricas para formas intermediárias a estáveis de dissolução, com perda da simetria dos planos de crescimento através da modificação progressiva da morfologia externa, iniciando da borda para o centro da face. Outras evidências deste fenômeno estão relacionadas à formação de feições na superfície dos diamantes, oriundas dos processos de dissolução química (Kukharenko 1954, Orlov 1966).

As relações entre a morfologia externa dos cristais de diamante (incluindo as feições de superfície) e as condições de formação têm sido objeto de estudos por mais de dois séculos. Kukharenko (1954) reconheceu a influência dos processos de crescimento e dissolução nos diamantes como oscilações nas condições dinâmicas presentes no manto e/ou na crosta terrestre, cujo sentido de deslocamento da reação crescimento?dissolução é função do equilíbrio físicoquímico característico para determinados processos. Kukharenko (1954) determinou as relações goniométricas de mais de 200 cristais de diamantes

1 Geoaktivan Ltda. - maximilianomartins@yahoo.com.br

${ }^{2}$ Instituto de Geologia da República de Komi - Syktvykar, Filial Uraliana da Academia de Ciências de Moscow, Rússia

${ }^{3}$ Depto. Geologia - IGC, Universidade Federal de Minas Gerais 
oriundos da região dos Montes Urais (Rússia), aos quais foram relacionadas as seguintes condições genéticas:

- formas de crescimento:

1) octaedros com textura lamelar, octaedros agrupados, cristais com forma esqueletal: evidenciam cristalização rápida a partir de um meio altamente supersaturado em carbono;

2) octaedros regulares: baixo grau de supersaturação em carbono;

3 ) formas combinadas: processo lento de cristalização sob condições saturadas de carbono.

- formas de dissolução:

4) octahedroids: formas remanescentes de dissolução lenta em meio subsaturado em carbono;

5) dodecahedroids: forma estável de dissolução em condições próximas às do equilíbrio de sistemas subsaturados;

6) trisoctaedros com arestas curvas e relevo alto: relacionadas à dissolução rápida seguida de combustão (queima);

7) formas irregulares com cavidades também irregulares: originadas a partir da corrosão e dissolução contínua seguida de queima;

8) formas de regeneração: cristais arredondados tais como dodecahedroids com fragmentos tabulares de relevo alto (positivo) nas faces, caracteristicamente perto das arestas.

Orlov (1977) corroborou e aperfeiçoou os estudos pioneiros de Shafranovskii (1948) e Kukharenko (1954) sobre a origem dos diamantes de aspecto abaulado e com arestas curvas. Seguindo a mesma nomenclatura utilizada por Kukharenko (1954), Orlov (1977) empregou o sufixo oid para designar as formas cristalográficas de crescimento que sofreram os efeitos da dissolução (em maior ou menor grau): dodecahedroid, octahedroid, cuboid, tetrahedroid e formas indeterminadas ou geminações que apresentem morfologia externa abaulada. A exemplo dos trabalhos anteriores, o dodecahedroid é reconhecido como a forma estável de dissolução de octaedros e cubos, enquanto a dissolução em formas combinadas depende do arranjo cristalográfico das superfícies de crescimento.

A reabsorção dos diamantes ocorre principalmente devido ao aumento da $\mathrm{fO}_{2}$, causando arredondamento do diamante, impressão de figuras de dissolução na superfície do cristal, e/ou consumo total do diamante. Este processo pode ocorrer no manto ou durante o transporte do diamante à superfície, quando o mesmo entra em contato com o líquido transportador cujo conteúdo de voláteis e condições mais oxidantes promovem sua reabsorção e corrosão (Robinson et al. 1989).

Salvo sob condições oxidantes (corrosivas), os processos de dissolução podem ser divididos naqueles que ocorrem sob temperaturas baixas, $<950^{\circ} \mathrm{C}$, e altas (Robinson 1980). A reabsorção a temperaturas elevadas promove a impressão de figuras de superfície e o desgaste das arestas de cristais cúbicos e octaédricos. A reabsorção a alta temperatura pode ocorrer no magma, entre 80 e $100 \mathrm{~km}$ de profundidade, por ação de $\mathrm{CO}_{2} \mathrm{e}$ vapor antes da cristalização da matriz. Diamantes inclusos em xenólitos mantélicos são preservados da reabsorção (McCandless et al. 1994). Aqueles parcialmente expostos podem ser em parte reabsorvidos e corroídos e são denominados pseudo-hemimorfos. Texturas como figuras de corrosão negativas foram consideradas típicas para a reabsorção a altas temperaturas e hexágonos, esculturas de corrosão, superfícies quimicamente polidas e frosting grosso também necessitariam de temperaturas maiores que $950^{\circ} \mathrm{C}$ (condições não oxidantes).

Sob temperaturas mais baixas (não oxidantes), inferiores a $950^{\circ} \mathrm{C}$, texturas com figuras de corrosão positivas são formadas. Entretanto, essas texturas não são comuns, atestando a raridade de reabsorção a baixas temperaturas.

Além das condições do magma favoráveis ou não à reabsorção durante o transporte, dois fatores são relevantes neste processo (Robinson 1980): 1) a profundidade em que o diamante é liberado do xenólito e 2) o tamanho do diamante. Neste sentido, uma pedra grande terá mais chances de alcançar a superfície (na forma reabsorvida) se comparada a uma menor quando liberadas na mesma profundidade devido à relação entre área superficial e volume. Em geral, a proporção de diamantes reabsorvidos aumenta com o decréscimo do tamanho das pedras em uma população. Apesar da taxa de reabsorção ser independente do tamanho da pedra, o baixo índice de preservação das pedras menores devese à elevada razão área superficial/massa.

Baseando-se em Robinson (1980) e Otter \& Gurney (1989), McCallumet al. (1994) e Otteret al. (1994) propuseram um esquema de classificação da morfologia externa de diamantes de acordo com o grau de preservação do cristal pela dissolução. Foram definidas seis classes de classificação entre cristais que apresentam pouca ou nenhuma evidência de dissolução (octaedros, cubo-octaedros ou cubos), até cristais com modificação completa da forma por estes processos, sendo o tetrahexahedroid designado como forma estável de reabsorção. Contudo, as relações goniométricas anteriormente realizadas por Kukharenko (1954) e Orlov (1977) para as faces do tetrahexahedroid atestam que esta forma constitui uma forma intermediária entre planos de crescimento de pseudo-tetraedros e dodecahedroids (Figura 1).

Analisando a morfologia externa e as feições de superfície de diamantes da região de Yakutia (Rússia), Evdokimov et al. (2001) reconheceram que mais de um processo de crescimento e dissolução podem ocorrer em um mesmo cristal, exibindo exatamente as mesmas feições de superfície, podendo representar estágios superpostos de crescimento e/ou dissolução. Assim, estruturas do tipo hillocks, trigons, e lamination lines não podem ser consideradas isoladamente como feições típicas de dissolução e/ou crescimento. Baseando-se nos trabalhos de Kukharenko (1954), Uruovskaya \& Orlov (1964) e Orlov (1977), Evdokimov et al. (2001) advogaram que a correta determinação da morfologia 


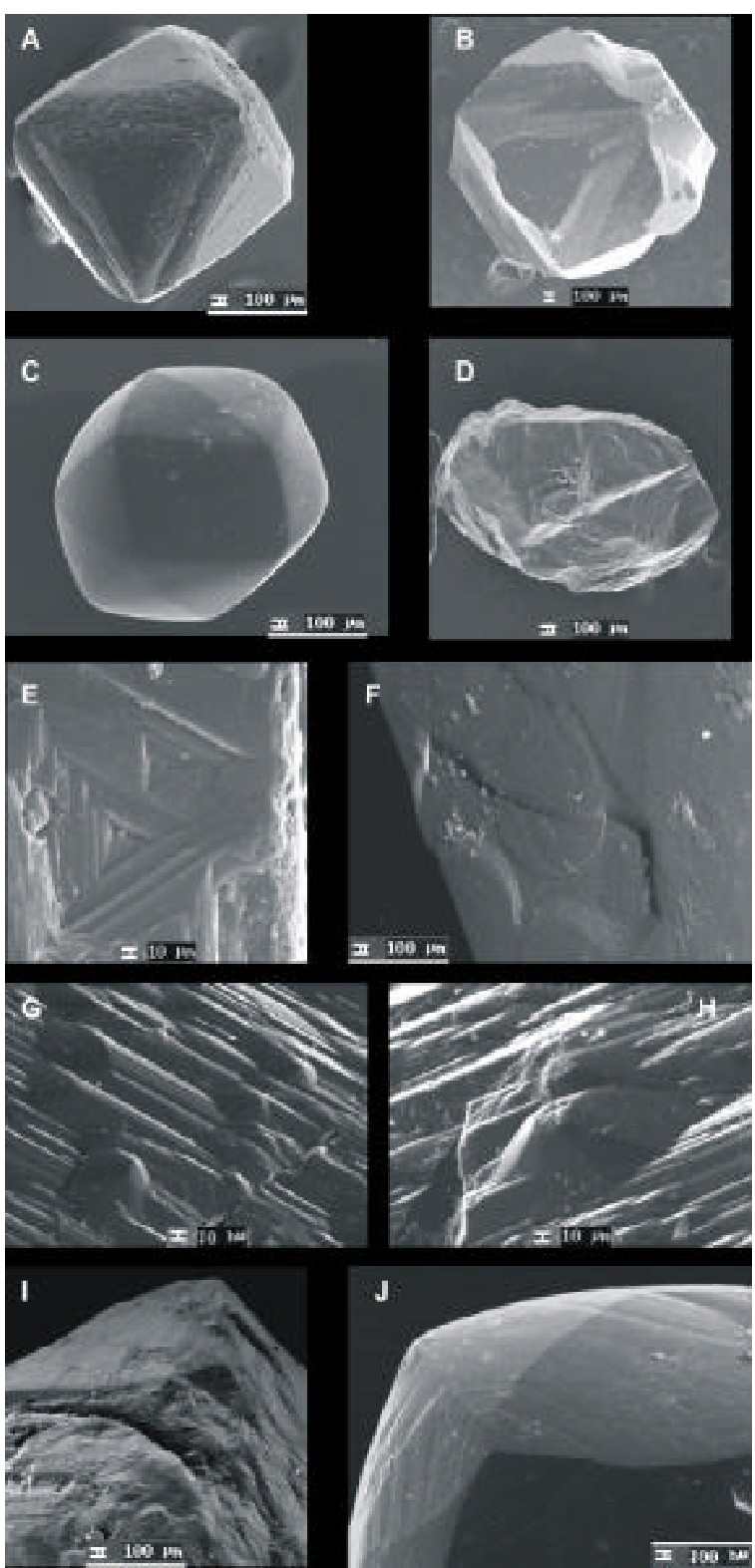

Figura 1- Morfologia externa e principais elementos de superficie nos diamantes da bacia do rio Macaúbas (Martins 2006):

(A) Octaedro com simetria preservada,

(B) transição octaedro-rombododecaedro,

(C) Rombododecaedro de faces lisas e arestas abauladas, (D) cristal irregular,

(E) sistemas de trigons com relevo negativo numa superfície clivada, $(F)$ estrutura em disco,

$(G)$ hillocks: em blocos e $(H)$ hillocks em pirâmide,

(I) canal de corrosão, (J) lamination lines com superfície de baixo relevo.

externa dos diamantes e das figuras de superfície oriundas dos processos de crescimento e/ou dissolução só é possível através das projeções goniométricas das superfícies dos cristais.

Com este propósito, foram realizados estudos sobre os padrões de reflexão na superfície de 47 diamantes da bacia do rio Macaúbas (MG) através do método da fotogoniometria (Rakin et al. 2004, Martins 2006).

\section{METODOLOGIA}

A fotogoniometria consiste na aplicação direta da Lei de Bragg para difração de raios laser (fonte monocromática) pela superfície dos diamantes. Foi feita a correção da Lei de Bragg para superfícies curvas a partir das relações trigonométricas entre as superfícies planas e curvas (Figura 2):

- Lei de Bragg para superfícies planas: $\hat{\mathbf{a}}=\operatorname{arc} \operatorname{tg}(\ddot{\mathbf{e}} /$ 2n ó1) + arctg (ё/2n ó2)

- Para superfícies curvas: $\mathbf{2}(\mathbf{R}-\mathbf{h}) / \mathbf{L}=$ ctgá

Os padrões de interferência emitidos pela superfície de cada cristal foram "capturados" em um papel fotográfico posicionado à frente do diamante afixado no goniômetro semi-circular, permitindo que o papel fotográfico velado exibisse exatamente os valores angulares de interferência desta superfície. Todos os cristais foram orientados segundo eixo [100] do cubo.

Uma vez que a Lei de Hauy estabelece que as relações entre os índices millerianos h, k e 1 (k/l e k/h) sejam sempre números racionais, decorre que os índices de Miller não determinam um plano quanto a sua posição absoluta, mas quanto a sua direção (Assunção 1964). Para se determinar os índices de Miller de cada face e subface, a imagem (papel fotográfico) foi escaneada e tratada no programa "Corel Xara", onde foi reticulada (escala de referência) e rotacionada, de modo que os eixos de simetria sejam diagonais ao centro de referência de rotação do cristal, tornando possível o cálculo dos índices de Miller de cada face e sub-face oriundas dos processos de crescimento ou dissolução.
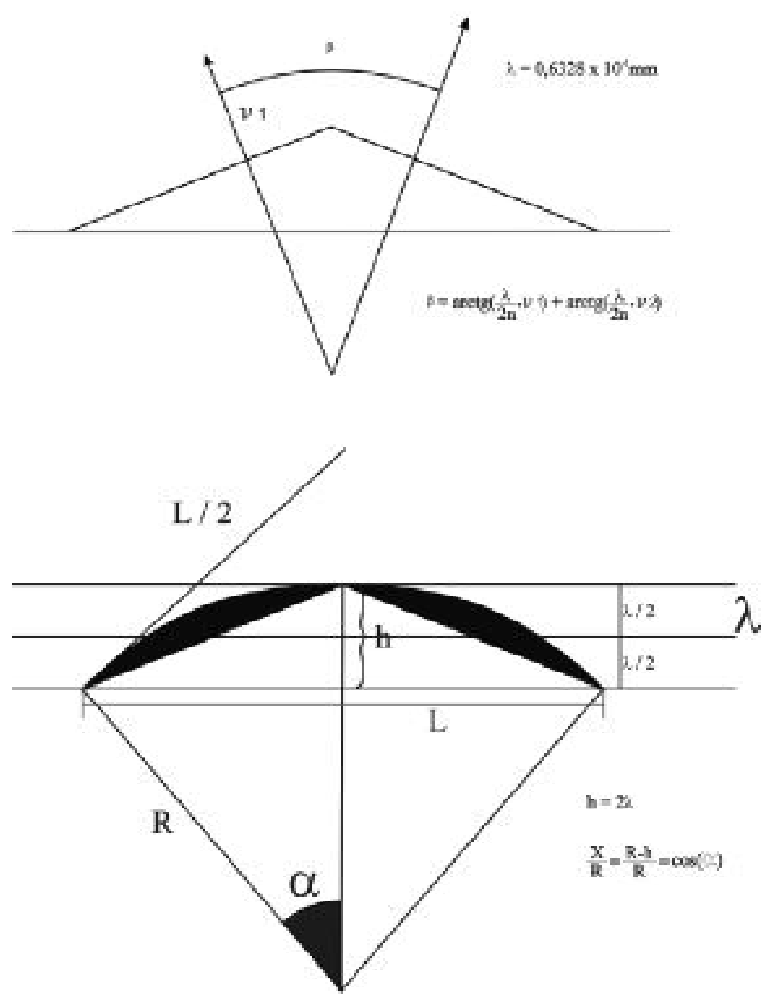

Figura 2 - Desenho esquemático dos padrões de interferência de superfícies planas e curvas segundo a Lei de Bragg. 
Análises detalhadas dos elementos de superfície de parte dos diamantes e carbonados foram realizados através do microscópio eletrônico de varredura (MEV) do Instituto de Geologia de Komi-Syktvykar (Rússia). O equipamento usado é do tipo Jsm-6400, dotado de sistema de dispersão de energia (EDS), prefixo Link Isis$300(\mathrm{U}=20 \mathrm{kV}, \mathrm{I}=1 \mathrm{nA}, \mathrm{t}=50 \mathrm{~s})$, calibrados através de metais puros ou óxidos. As superfícies das amostras foram limpas com acetona usando-se um dispersor ultrasônico, sendo posteriormente metalizadas com grafite. Áreas planas foram selecionadas para análises químicas de inclusões nos carbonados, cuja acuracidade foi checada pela soma dos componentes e pela repetição das análises em diferentes pontos da amostra (TEM, modelo Tesla Bs-500).

\section{RESULTADOSOBTIDOS POR FOTOGONIOMETRIA EDISCUSSÕES}

Levando-se em consideração a locação dos átomos de carbono na célula elementar, a estrutura de simetria do diamante é definida pelo grupo $43 \mathrm{~m}$. Contudo, pela morfologia, as faces de formas simples $\{111\}$ e $\{111\}$ não diferem uma da outra. Os coeficientes de tensão das faces octaédricas como propriedades física dos diamantes indicam um alto grau de simetria m3m, incluindo, pelo Princípio de Nairman, o grupo 43m. Desta forma, de acordo com a morfologia, a simetria do cristal crescendo num meio isotrópico pode ser considerada como $\mathrm{m} 3 \mathrm{~m}$, mas a dissolução atuando isotropicamente no cristal não poderia decrescer com simetria externa $\mathrm{m} 3 \mathrm{~m}$. Através dos resultados de reflexão de diamantes naturais por goniômetro parabólico, estabeleceu-se que os complexos arranjos de reflexão de superfícies curvas podem ser divididos em três pontos (segundo os grupos $4 / \mathrm{m}, 2 / \mathrm{m}$ e $\overline{6}$, Kukharenko 1954).

Pelo Princípio de Curie, o decréscimo inicial do grupo de simetria cristalográfico m $3 \mathrm{~m}$ para 4/m, 2/m e $\bar{\sigma}$ pode ser explicado pela rotação do cristal em um ambiente homogêneo não-saturado. Supondo, para o caso mais simples, que a dissolução do cristal ocorre ao mesmo tempo que a rotação do cristal ao redor dos eixos de rotação, então o eixo de rotação coincidirá com os eixos de simetria de $4^{\mathrm{a}}$ ordem e o grupo de simetria decrescerá até para 4/m. Entre os 3 eixos de simetria de L4, apenas um permanecerá, coincidindo com o eixo de rotação, enquanto os outros eixos de simetria se degenerarão em planos de simetria. Quando o eixo de rotação do cristal coincidir com os eixos de $2^{\text {a }}$ ordem da estrutura, a simetria decrescerá a $2 / \mathrm{m}$, e a rotação ao redor do eixo de $3^{a}$ ordem resultará no decréscimo da simetria externa até $\overline{6}$, correspondentemente.

Como resultado do cálculo dos limites das projeções parabólicas pelo Algoritmo de Varoney, controlado pelos eixos de $4^{\mathrm{a}}, 3^{\mathrm{a}}$ e $2^{\mathrm{a}}$ ordens, os valores correspondentes para a freqüência de ocorrência são $28 \%, 28 \%$ e $44 \%$. Desta forma, a probabilidade de identificar os reflexos de superfícies curvas em cristais dissolvidos através de projeções cristalográficas é de 0.28 para o grupo $4 / \mathrm{m}, 0.28$ para o grupo $\overline{6}$ e de 0.44 para o grupo $2 / \mathrm{m}$.

Para a descrição das faces curvas dos diamantes, primeiramente é determinada a morfologia dos cristais, sendo as feições de superfícies classificadas com maior detalhe.

De acordo com Orlov (1977), os valores das distâncias angulares entre as figuras de reflexão conservam as formas características para cristais arredondados pela dissolução devido às formas triangulares de reflexão a partir das superfícies elementares de cristais de arestas e superfícies curvas. De acordo com os resultados dos cristais estudados por dois goniômetros circulares, 3 feições particulares podem ser correlacionadas àquelas determinadas por Shafranovskii (1948): dodecahedroid, octahedroid e cuboid (Tabela 1, Figura 3).

Contudo, os valores dos ângulos de reflexão em projeção estereográfica não podem ser indistintamente associados a superfícies curvas com suas formas, correspondentemente.

A principal necessidade de se determinar a aparência dos cristais com nenhuma ou pouca dissolução deve-se à localização dos máximos de intensidade no campo das reflexões das projeções cristalográficas. Três pontos próximos ao centro goniométrico de reflexão determinam cristais dodecaédricos (110), octaédricos (111) e tetrahexaédricos (210). Desta forma, é assumido o

Tabela 1 - Parâmetros de reflexão de diamantes com superfície curva. Dados retirados de Orlov (1977).

\begin{tabular}{|c|c|c|c|c|c|}
\hline \multicolumn{2}{|c|}{ Dodecahedroid } & \multicolumn{2}{c|}{ Octahedroid } & \multicolumn{2}{c|}{ Cuboid } \\
\hline Distância & Ângulo $\left(^{\circ}\right)$ & Distância & Ângulo $\left(^{\circ}\right)$ & Distância & Ângulo $\left(^{\circ}\right)$ \\
\hline AB & 36.07 & AB & 62.43 & AB & 25.30 \\
\hline D'C' & 13.15 & D'C' & $?$ & D'C' & $?$ \\
\hline D'D” & 13.15 & D'D" & 6.39 & D'D” & $?$ \\
\hline C'C' & 39.37 & C'C' & 47.29 & C'C' & 61.05 \\
\hline
\end{tabular}




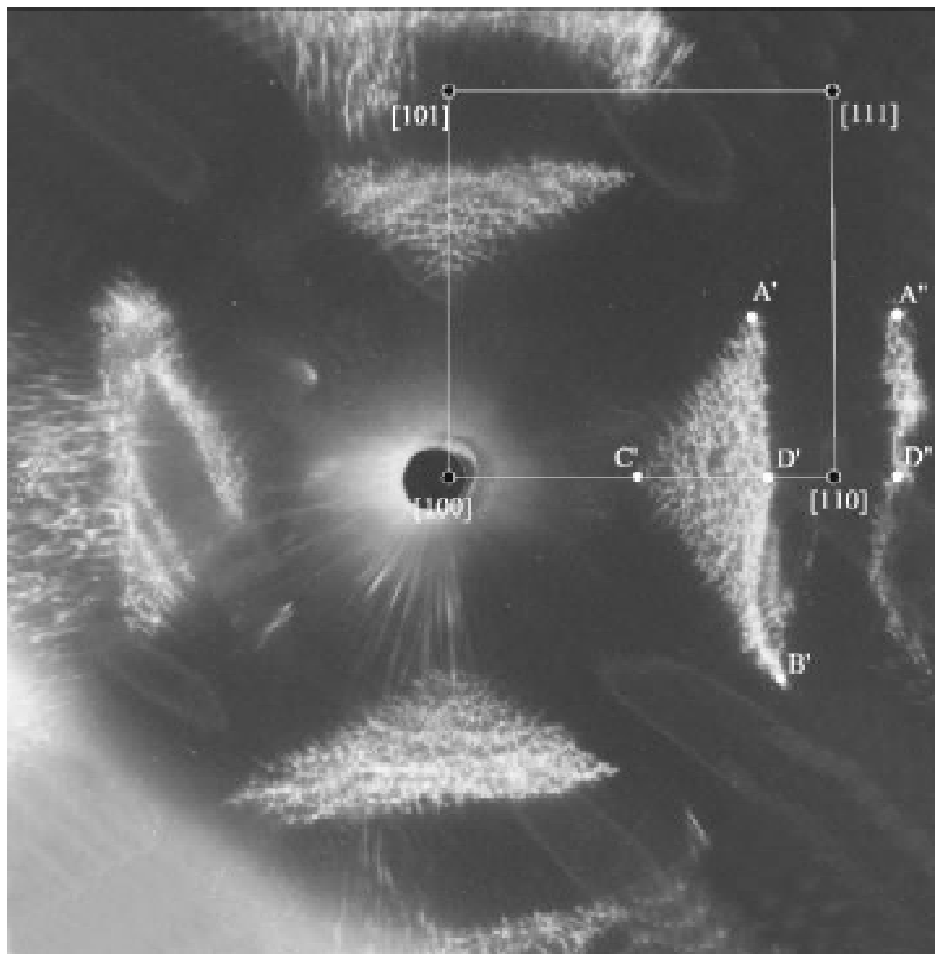

Figura 3 - Fotogonomograma de um diamante do tipo dodecahedroid orientado ao longo de L4, evidenciando a perda de planos de simetria paralelos aos eixos, com decréscimo da forma de simetria do cristal até $4 / \mathrm{m}$. Pontos característicos de reflexão são distinguidos.

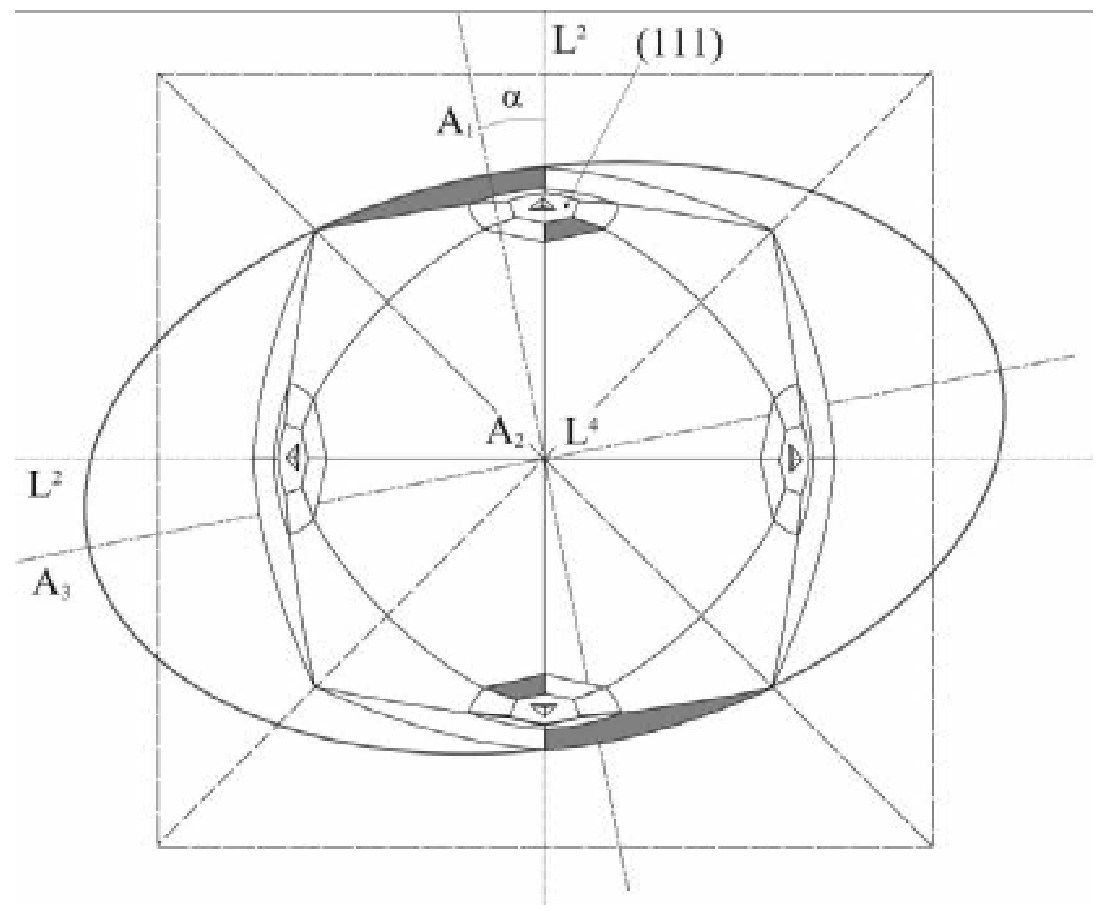

Figura 4 - Forma elipsoidal de um cristal arredondado, inicialmente octaédrico (linhas pontilhadas). As superfícies pertencentes ao elipsóide são marcadas por cores escuras. 
seguinte pressuposto: quanto maior a área de reflexão em projeção goniométrica de uma superfície curva (Figura 4), mais dissolvido e arredondado será o cristal.

Como resultado do estudo das formas dos diamantes no goniômetro parabólico, estabeleceu-se que a superfície curva nos dodecahedroids podem ser descritas pelos 3 eixos elipsoidais regularmente posicionados em relação à estrutura do cristal e multiplicados pelo grupo de simetria $\mathrm{m} 3 \mathrm{~m}$ até 12 (Figura 4). O elipsóide com semi-eixos A1, A2 e A3 (numerados em ordem crescente) está orientado com relação à estrutura do cristal. Desta forma, o semi-eixo A2 estará sempre orientado ao longo do eixo L4, enquanto os outros dois semi-eixos estarão associados ao eixo L2, perpendicular ao eixo de $4^{\mathrm{a}}$ ordem e de menor ângulo (á, Figura 4). A interseção de superfícies simétricas no elipsóide de projeção resultará numa superfície caracterizada pela reflexão triangular dos parâmetros ABC (Figura 3). Pequenas superfícies não-simétricas são estipuladas pela direção de rotação do cristal como descrito anteriormente. O total de superfícies curvas limitando o dodecahedroid é igual a 24 .

A forma da superfície do diamante relacionado ao elipsóide de projeção evidencia o caráter dinâmico da estabilidade do mineral frente aos processos de dissolução e de abrasão mecânica. Dois entre quatro eixos de simetria do eixo L3 são paralelos ao semi-eixo A1, quase sempre normal à superfície do elipsóide e que registram formas reliquiares dos estágio iniciais de dissolução. É muito importante que tais formas estejam localizadas nos centros das faces de octaedros perfeitos, coincidindo com as seis superfícies adjacentes ao elipsóide. Esta é a razão pela qual a face de um octaedro possui grande estabilidade frente à dissolução, podendo ser encontrado em forma reliquiar. Em tais situações, aparecerão pontos de aquecimento térmico nas faces octaédricas. Estes pontos registram um processo lento de dissolução difusa características para superfícies curvas iguais à zero ou negativas.

Com a presença de faces reliquiares octaédricas em um cristal, podem ser encontrados ao redor de seis fragmentos de superfícies pertencentes ao elipsóide (Figura 3). Dois reflexos característicos no gonomograma (Figura 5), delimitados pelos vértices [100], [110] e [111] foram interpretados anteriormente como duas formas diferentes de dissolução: dodecahedroid com face-seam (Orlov 1977) e trigohexahedroid (McCallum et al. 1991, 1994, Otter et al. 1994). Contudo, através dos padrões de reflexão obtidos no goniômetro, as formas de um elipsóide são reproduzidas por elementos de simetria. Esta é a razão para explicar o seguinte fato observado: as superfícies do trigohexahedroid são preservadas somente se existirem faces reliquiares adjacentes ao octaedro (figuras 6 e 7). Considerando-se as faces octaédricas reliquiares e a razão entre os semi-eixos A2/A1, pode-se observar mais de oitenta superfícies planas e convexas separadas por arestas vivas.

De acordo com as reflexões do cristal (vide Figura 4), pôde-se determinar os parâmetros que podem definir a forma do elipsóide (A1, A2, A3) e os valores angulares usando as seguintes fórmulas:

$$
\begin{aligned}
& A 1=d / 2, \\
& A 2=A 1 v \operatorname{tg} \delta / \operatorname{tg} \beta, \\
& A 3=v \operatorname{tg}(\varepsilon-a) / \operatorname{tg} \gamma),
\end{aligned}
$$

onde $\mathrm{d}=$ diâmetro do cristal na direção do semi-eixo $\mathrm{A} 1, \bar{\delta}=35.26^{\circ}$ (ângulo entre as direções [111] e [110]); $\varepsilon=45^{\circ}$ (ângulo entre as direções [100] e [110]), y= ângulo entre os pontos $\mathrm{C}$ e $\mathrm{D}$.

As fórmulas anteriormente apresentadas são calculadas considerando-se os menores ângulos, as fórmulas aproximadas e idealizadas do dodecahedroid com face-seam (sem faces reliquiares dodecaédricas). $\mathrm{Na}$ prática, utilizam-se comumente os valores dos comprimentos dos semi-eixos relacionados ao semi-eixo A1 (menor dos semi-eixos). Usando-se as projeções da dissolução no elipsóide, é possível descrever o desenvolvimento da forma do cristal desde o octaedro original e todas as modificações ocorridas durante o processo de dissolução. Os números de dissoluções observadas podem ser graficamente representada pelas projeções goniométricas normais à superfície do cristal (Figura 7).

O início da dissolução pode resultar na formação de superfícies cilindro-elípticas (elipsóide com parâmetros A1/A1, A2/A1, A3/A1, á? 1, 1.4, $\infty, 0^{\circ}$ ) (figuras 7A e 7B). Nas faces do octaedro, pontos de aquecimento térmico desenvolvem-se, aos quais possuem subfaces paralelas ao cilindro-elíptico. Esta é a explicação para a orientação inversa (anti-paralela) dos pontos de aquecimento térmico ao lado das superfícies que são marcadas por três pontos na projeção goniométrica ao redor dos refluxos da face octaédrica (111). Posteriormente, durante a evolução do processo de dissolução, a face-seam aumenta progressivamente, indicando a inversão do cilindro elíptico em dois elipsóides simétricos com ângulo de rotação á (Figura 7C). De acordo com as medidas realizadas no presente trabalho e com os valores obtidos por Orlov (1977), este ângulo atinge $7^{\circ}$ no limite próximo às arestas do cristal que ainda possuem forma octaédrica concomitantemente com o aparecimento de superfícies de trigohexahedroids ao redor das faces do octaedro. As superfícies do elipsóide, juntamente com as do trigohexahedroid, geram as superfícies orientadas do elipsóide, marcadas pelas linhas pontilhadas (Figura 7D). Durante a dissolução posterior, fragmentos das superfícies do dodecahedroid e do trigohexahedroid tornam-se mais convexas (Figura 7E). A dissolução progressiva implica no desaparecimento das faces do octaedro e do trigohexahedroid e somente as superfícies do dodecahedroid com face-seam preservam-se, produzindo os reflexos característicos nas projeções goniométricas (Figura 7F).

Durante a dissolução, os parâmetros dos semieixos do elipsóide das superfícies do cristal tornam-se 


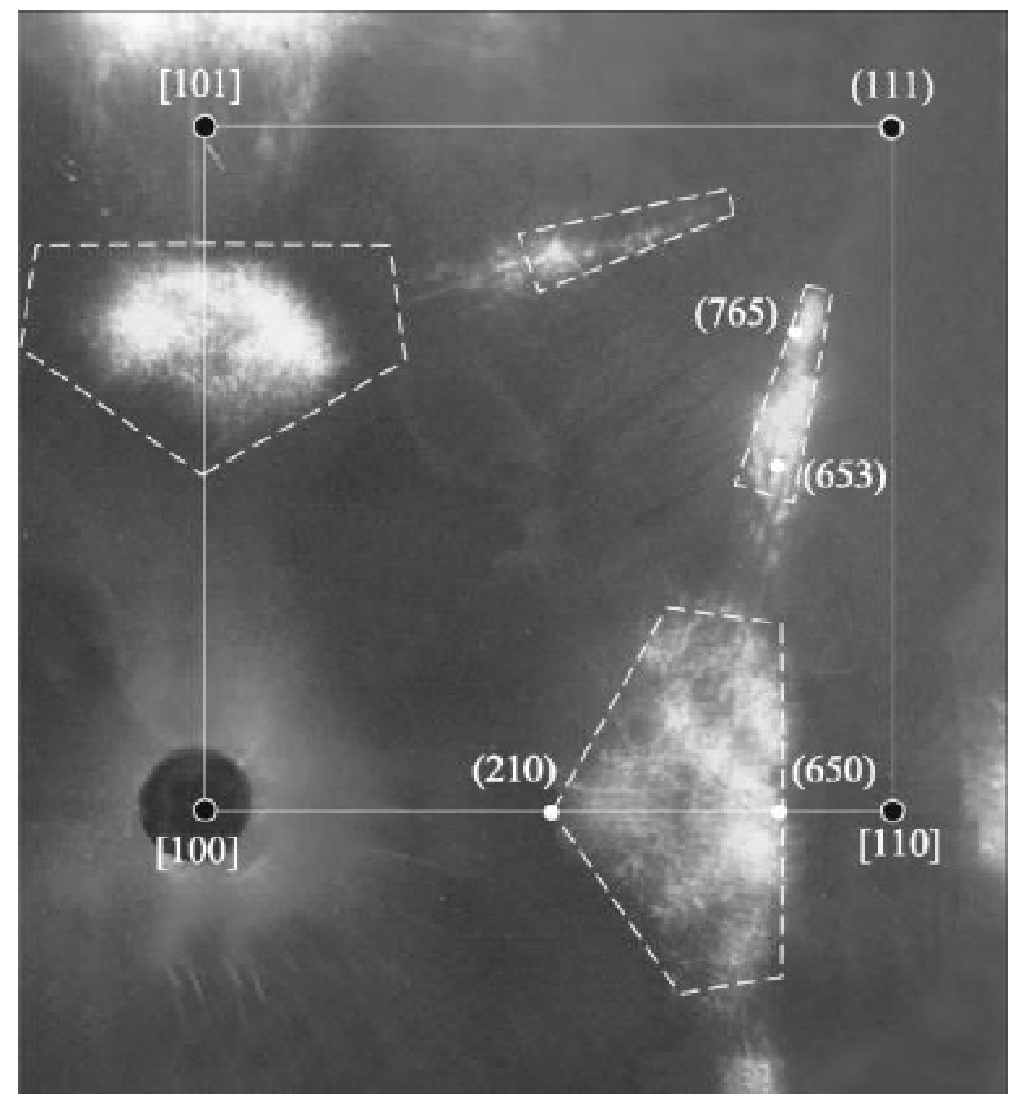

Figura 5 - Fotogonomograma de um diamante com superfícies convexas do dodecahedroid e trigohexahedroid (distinguido pelas linhas tracejadas) pertencendo ao elipsóide simétrico por um conjunto de parâmetros.

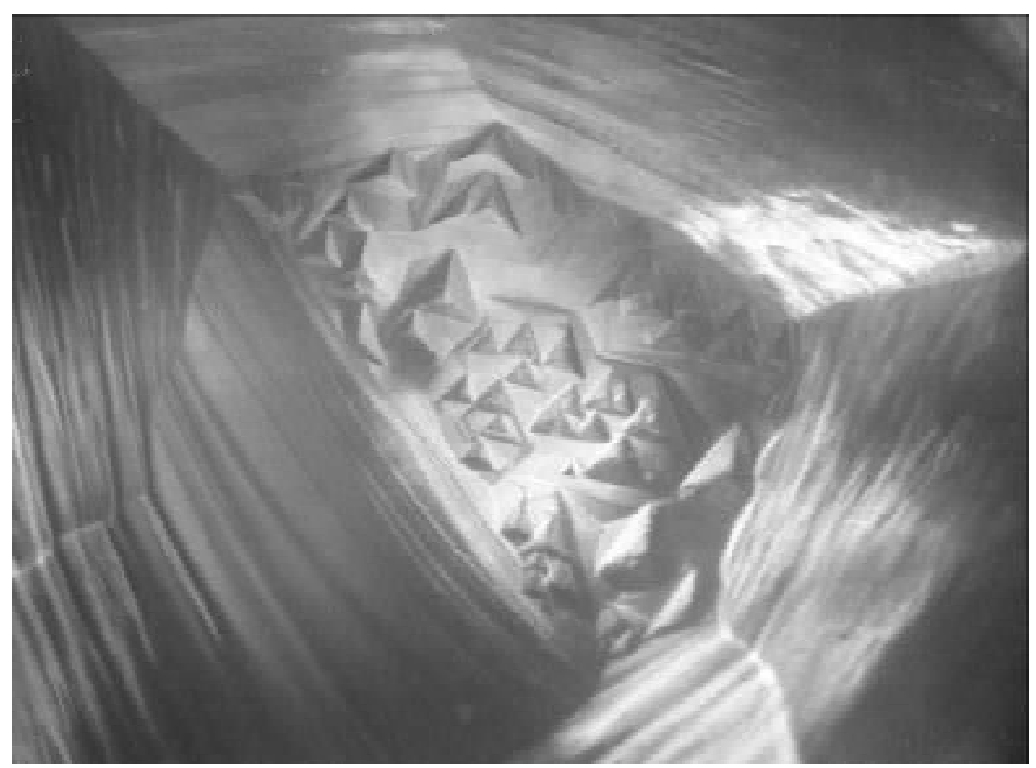

Figura 6 - Superfície de dissolução com fragmento reliquiar da face octaédrica "decorada” por "pontos" de aquecimento térmico orientados anti-paralelos à face, dodecahedroid e trigohexahedroid separados por arestas. 
A)

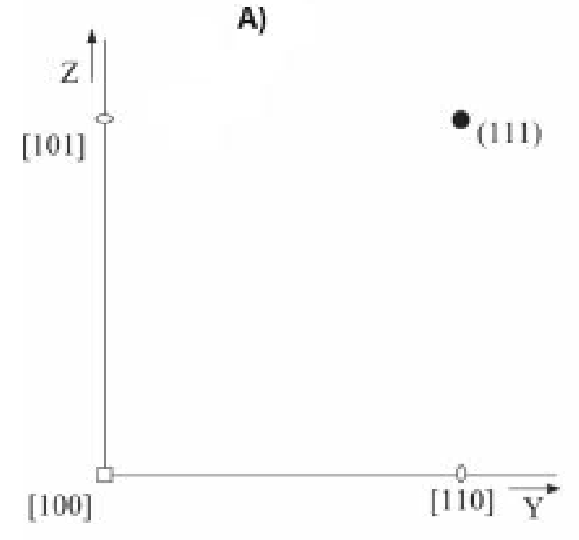

c)

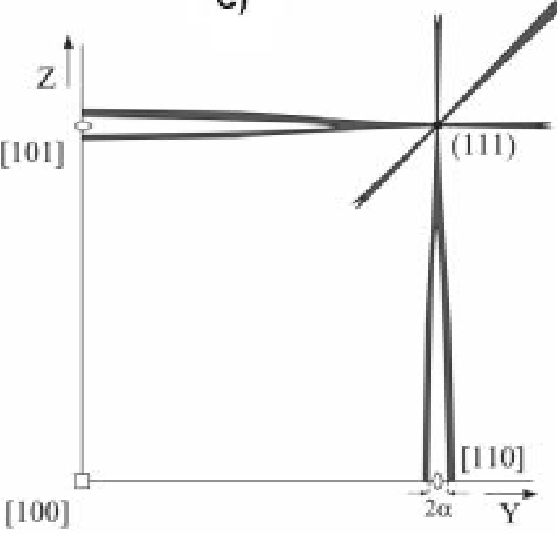

E)

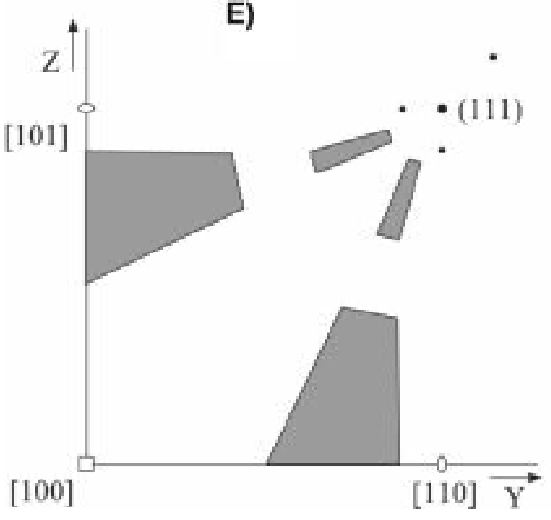

B)

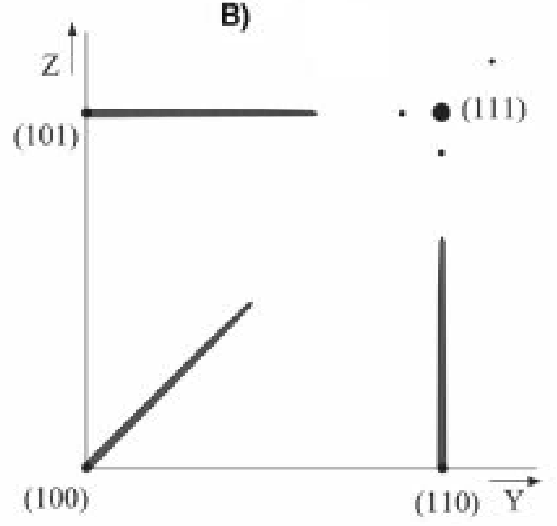

D)

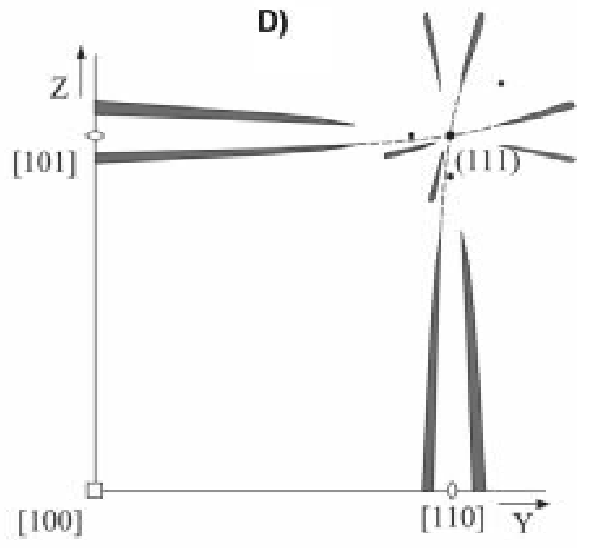

F)

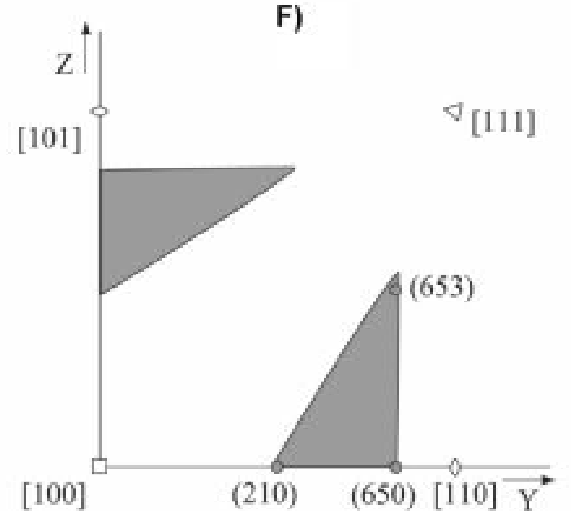

Figura 7 - Evolução da dissolução no diamante: A) estágio inicial - octaedro perfeito;

B) início da dissolução - formação de um cilindro elíptico; C) formação da face-seam;

D) início da formação das superfícies do trigohexahedroid; E) desenvolvimento das superfícies convexas do dodecahedroid (com face-seam) e trigohexahedroid; F) forma limite de um dodecahedroid com face-seam. Gonomogramas sumarizados das superfícies do primeiro quadrante. Superfícies atuais marcadas a cores, símbolos das direções são marcadas por polígonos vazios.

menores, ao mesmo tempo que as superfícies preservadas dos pontos de aquecimento térmico nas faces octaédricos reliquiares mostram padrões de reflexão no elipsóide, característico para os estágio iniciais do processo. Isto é explicado pelo lento mecanismo de difusão da dissolução em superfícies com curvatura igual a zero ou negativa, diferente do mecanismo "abrasivo" sobre superfícies complexas.
Quatro faces com pontos de aquecimento apresentamse sempre regulares, formadas pelas superfícies do cilindro elíptico nas superfícies dos cristais arredondados, localizados próximos às extremidades dos eixos de $4^{\mathrm{a}}$ ordem. Todos os 47 diamantes da bacia do rio Macaúbas apresentam uma história de dissolução prolongada, caracterizados por dodecahedroids com os seguintes parâmetros do elipsóide: $1^{\circ}, 1.23^{\circ}, 1.41^{\circ} \mathrm{e} 6.3^{\circ}$. 
É interessante notar que mesmo insignificantes fenômenos de regeneração nos diamantes (Figura 8) praticamente mudam o padrão de reflexão das superfícies parabólicas goniométricas, evidenciado pelo desaparecimento de grandes áreas claras e pelo aparecimento de pontos luminosos nas posições octaédricas (111). Desta forma, é fácil o estabelecimento de feições de regeneração nos diamantes: cristais com qualquer forma produzem uma combinação polar de octaedros perfeitos com superfícies de regeneração na tela de projeção goniométrica.

Múltiplas feições de regeneração são estabelecidas nos cristais arredondados (figuras 8 e 9). É evidente que sucessivos processos de crescimento e regeneração nos diamantes ocorrem em condições mantélicas. De uma forma geral, depois do estágio de regeneração, um próximo estágio de dissolução poderá ocorrer e a superfície do cristal apresentará uma estrutura complexa com hillock e textura shagreen paralelas a feições de dissolução. Também a presença de formas rasas dos pontos de aquecimento térmico paralelos à orientação das faces octaédricas é um indício de significantes processos de regeneração nos cristais, assim como faces octaédricas com buracos no lugar
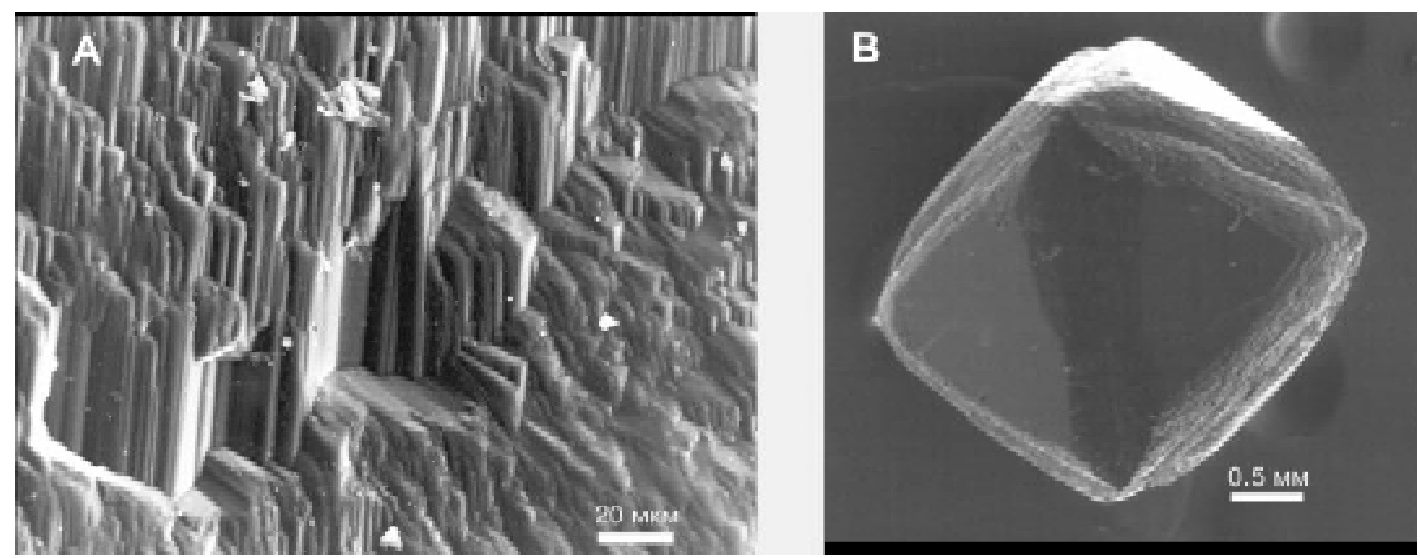

Figura 8 - Regeneração das superfícies do diamante com diferentes estágios: A) pequeno tempo de regeneração; B) longo tempo de regeneração de um cristal arredondado pela dissolução.

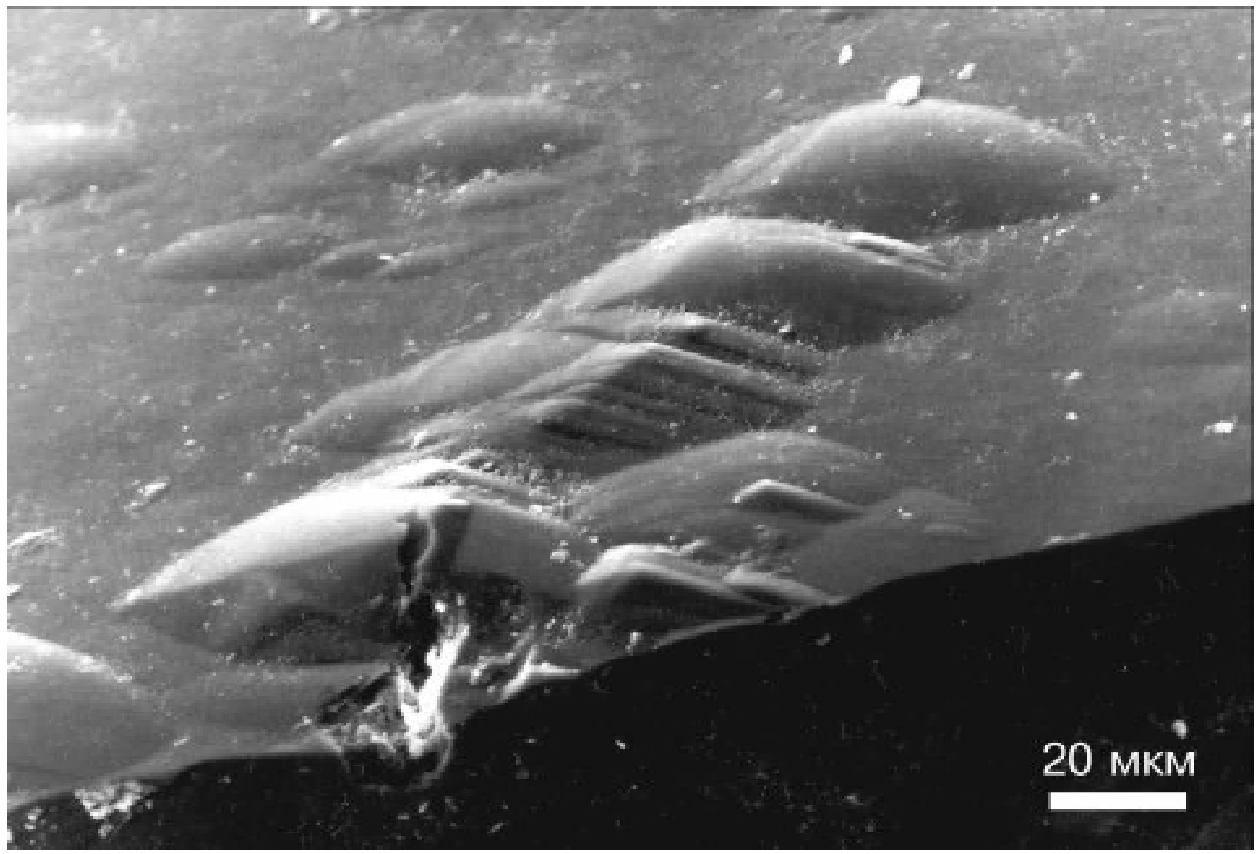

Figura 9 - Hillocks de regeneração na superfície de um diamante dodecahedroid. 
das arestas (110) (Figura 7B). Tais cristais crescem por um longo período de regeneração a partir de diamantes curvos com recobrimento incompleto de todo octaedro neo-formado.

\section{AGRADECIMENTOS}

M. Martins agradece ao Colegiado de Pósgraduação em Geologia - IGC/UFMG pela concessão da bolsa CAPES durante o período de realização da tese de doutoramento. Pelos auxílios financeiros e facilidades analíticas os autores agradecem ao Instituto de Geologia de Komi (Rússia), na pessoa do Acadêmico Dr. Nikholay P. Yuskin. Aos revisores da GEONOMOS.

\section{REFERÊNCIAS BIBLIOGRÁFICAS}

Assunção, C.T. 1964. Temas clássicos em cristalografia. Classes de simetria e formas simples. Soc. Portuguesa de Ciências Naturais, Coleção "Natura", 56p.

Bulanova, G.P. 1995. The formation of diamond. Journal of Geochemical Exploration, 53:1-23.

Evdokimov, M.D., Ladygina, M.Y., Nesterov, A.R. 2001. Morphology of diamonds as a possible indicator of their genesis. N. Jb. Miner. Abh., 176(2):153-177.

Helmstaedt, H.H. \& Gurney, J.J. 1995. Geotectonic controls of primary diamond deposits: implications for area selection. Journal of Geochemical Exploration, 53:125-144.

Kukharenko, A.A. 1954. On rounded diamond crystals. Uchem. Zap. Leningrad Gos. Univ., 178(4), 55p. (em russo).

Martins, M.S. (2006). Geologia dos diamantes e carbonados aluvionares da bacia do rio Macaúbas (MG). Tese de Doutoramento, IGC/UFMG, 248p (inédito).

McCallum, M.E., Huntley, P.M., Falk, R.W., Otter, M.L. 1991. Morphological, resorption and etch feature trends of diamonds from kimberlite populations within the Colorado Wyoming State line district, USA. In: CPRM/ Fifth international kimberlite conference, Araxá, Minas Gerais, Brasil. Vol. 2: Diamonds: characterization, genesis and exploration, p.32-50.
McCallum, M.E., Huntley, P.M., Falk, R.W., Otter, M.L. 1994. Morphological, resorption and etch feature trends of diamonds from kimberlite populations within the ColoradoWyoming State Line District, USA. In: Proceedings da V Conf. Intern. de kimb. (ed. H.O.A. Meyer e O.H. Leonardos). V.2 - Diamonds:characterization, genesis, and exploration. Araxá, Brasil:32-50.

McCandless, T.E., Waldman, M.A., Gurney, J.J. 1994. Macrodiamonds and microdiamonds from Murfreesboro lamproites, Arkansas: morphology, inclusions and carbon isotope geochemistry. In: CPRM/ Núcleo Brasília, Fifth international kimberlite conference, Araxá, Brazil. 2: 7897.

Meyer., H.O.A. 1985. Genesis of diamond. A mantle saga. American Mineralogist, 70:344-355.

Orlov, Yu. L. 1966. Evidence that the rounding of diamond is caused by dissolution process. Min. Sbor. Louvsk. Univ., 20:129-130.

Orlov, Yu. L. 1977. The mineralogy of diamond. Izdatel'stva Nanka. Translated from Russian Geology and Geophysics by WILEY,J. \& SONS. New York. 235 p.

Otter, M.L. \& Gurney, J.J. 1989. Mineral inclusions in diamonds from the Sloan diatremes, Colorado-Wyoming State Line district, North America. In: Proc. da V Conf. Intern. Kimb. Kimberlites and related rocks, 2:1042-1053.

Otter, M.L., McCallum, M.E., Gurney, J.J. 1994. A physical characterization of the Sloan (Colorado) diamonds using a revised diamond description scheme. In: CPRM/ Núcleo Brasília, Fifth international kimberlite conference, Araxá, Brazil. 2: 15-31

Robinson, D.N. 1980. Surface textures and other features of diamonds. Tese de Doutoramento, Natal, África do Sul, 221p.

Robinson, D.N., Scott, J.A., van Niekerk, A., Anderson, V.G. 1989. The sequence of events reflected in the diamonds of some southern African kimberlites. Geol. Soc. Australia. Sp. Publ., 14(2): 990-1000.

Rakin, V., Martins, M., Karfunkel, J. 2004. The shape of curvefaced diamonds. In: 32TH Intern. Cong. of Geology, Florence. CD-Rom.

Shafranovskii, I.I. 1948. On the crystallography of Braziliantype diamond. Dokl. An SSSR, 26(7) (em russo).

Uruovskaya, A.A. \& Orlov, Y.L. 1964. Nature of plastic deformation of diamond crystals. Doklady Akademii Nauk SSSR, 154:112-115 (em russo). 